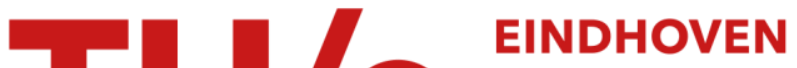 UNIVERSITY OF TECHNOLOGY
}

\section{An inverse dynamics modeling approach to determine the restraining function of human knee ligament bundles}

\section{Citation for published version (APA):}

Mommersteeg, T. J. A., Huiskes, H. W. J., Blankevoort, L., Kooloos, J. G. M., \& Kauer, J. M. G. (1997). An inverse dynamics modeling approach to determine the restraining function of human knee ligament bundles. Journal of Biomechanics, 30(2), 139-146. https://doi.org/10.1016/S0021-9290(96)00096-6

DOI:

10.1016/S0021-9290(96)00096-6

Document status and date:

Published: 01/01/1997

\section{Document Version:}

Publisher's PDF, also known as Version of Record (includes final page, issue and volume numbers)

\section{Please check the document version of this publication:}

- A submitted manuscript is the version of the article upon submission and before peer-review. There can be important differences between the submitted version and the official published version of record. People interested in the research are advised to contact the author for the final version of the publication, or visit the $\mathrm{DOI}$ to the publisher's website.

- The final author version and the galley proof are versions of the publication after peer review.

- The final published version features the final layout of the paper including the volume, issue and page numbers.

Link to publication

\section{General rights}

Copyright and moral rights for the publications made accessible in the public portal are retained by the authors and/or other copyright owners and it is a condition of accessing publications that users recognise and abide by the legal requirements associated with these rights.

- Users may download and print one copy of any publication from the public portal for the purpose of private study or research.

- You may not further distribute the material or use it for any profit-making activity or commercial gain

- You may freely distribute the URL identifying the publication in the public portal.

If the publication is distributed under the terms of Article 25fa of the Dutch Copyright Act, indicated by the "Taverne" license above, please follow below link for the End User Agreement:

www.tue.nl/taverne

Take down policy

If you believe that this document breaches copyright please contact us at:

openaccess@tue.nl

providing details and we will investigate your claim. 


\title{
AN INVERSE DYNAMICS MODELING APPROACH TO DETERMINE THE RESTRAINING FUNCTION OF HUMAN KNEE LIGAMENT BUNDLES
}

\author{
T. J. A. Mommersteeg, ${ }^{*} \dagger$ R. Huiskes, $\dagger \ddagger$ L. Blankevoort, $\dagger$ J. G. M. Kooloos* and J. M. G. Kauer* \\ *Department of Anatomy and Embryology, University of Nijmegen, The Netherlands; and $†$ Biomechanics \\ Section, Institute for Orthopedics, University of Nijmegen, P.O. Box $9101,6500 \mathrm{HB}$, Nijmegen, \\ The Netherlands
}

\begin{abstract}
During knee motion, the fiber bundles of ligaments are nonuniformly loaded in a recruitment pattern which is different for successive knee-joint positions. As a result, the restraining functions of these ligaments are variable. To analyze the relative restraint contributions of the fiber bundles in different knee-joint positions, a new method was developed. Its application was illustrated for the cruciate ligaments of one knee-joint specimen.

The methods developed to estimate bundle forces comprise five steps. First, the three-dimensional motions of a knee specimen are measured for anterior-posterior forces, using Röntgen Stereophotogrammetric Analysis. Second, bone-ligament-bone tensile tests are performed to evaluate the mechanical properties of these structures in several relative orientations of the bones. Third, multiple fiber bundles are identified in each ligament, based on the main fiber orientations. Fourth, the nonlinear force-length relationship of each functional bundle, as defined by a stiffness and a recruitment parameter, is determined by combining the multidirectional tensile tests with a multiline-element ligament model. Finally, the information obtained is combined in a whole-joint computer model of the knee, to determine the internal forces in the initial kinematic experiment, using an inverse dynamics approach.

The technique appeared to be extremely time consuming and technologically involved. However, it was demonstrated to be useful and effective. The preliminary results reveal that the fiber bundle restraints are extremely sensitive to the knee flexion angle and the restraining forces are highly variable within the ligaments. For both cruciate ligaments, a gradual transition was demonstrated in load transfer from the posterior bundles to the more anteriorly positioned ones during knee flexion. Furthermore, it appeared that relatively high forces were carried by only a few fiber bundles at each flexion angle. Based on these preliminary results, it is concluded that the determination of forces in multiple ligament bundles is important for the understanding of failure mechanisms of ligaments. In particular, alternate loading of different fiber bundles suggests that successful operative reconstruction of the cruciate ligaments may not be achieved simply by a one-bundle preparation. Copyright (C) 1996 Published by Elsevier Science Ltd.
\end{abstract}

Keywords: Knee model; Knee ligaments; Ligament bundles; Function.

\section{INTRODUCTION}

Human knee ligaments are geometrically complex and mechanically nonhomogeneous tissues. As an effect of these characteristics, the loading patterns of these structures are typically three-dimensional in nature: they transfer loads nonuniformly, in several directions, displaying variable load-displacement characteristics during knee motions. In this way, in perfect tuning with other knee structures, they are able to provide the knee with sufficient stability and movability. Understanding these complex mechanisms underlying the function of knee ligaments is important for the evaluation of partial ligament ruptures as well as for the design of ligament replacements.

The role the ligaments play in knee mechanics has been studied by the measurement of ligament forces, using a variety of methods. Direct measurements of forces in the four major ligaments of the knee were performed in situ using buckle transducers (Lewis et al., 1982, 1989; Ahmed et al., 1987, 1992). Selected bundles of knee ligaments were instrumented and the loads in two

Received in final form 29 May 1996.

$\ddagger$ Author to whom correspondence should be addressed. flexion angles of the joint measured. To obtain whole ligament forces, Markolf et al. $(1991,1993)$ and Wascher et al. (1993) connected a load-cell to the tibial insertion sites of the anterior and posterior cruciate ligaments of the knee. These studies have provided considerable information concerning the in situ forces developed within the cruciate ligaments. In order to determine the restraining functions of the ligaments, however, the directions of the forces relative to the externally applied forces have to be taken into account. In recent studies, the functions of the anterior cruciate ligament and its major bundles in restraining an anterior tibial force of $100 \mathrm{~N}$ were determined by selective cutting (Blomstrom et al., 1993; Guan et al., 1991). In these studies the load-transmitting role of the removed structure was determined. A disadvantage of this approach is that the removal of (part of) a structure could influence the loading patterns of the remaining knee structures as an indirect effect.

An alternative to direct measuring techniques is the indirect assessment of ligament forces from inverse dynamics models (Huiskes, 1992). If the positions of the ligament insertion sites are known relative to the geometry of the bones, and the force-displacement characteristics of the ligament as well, the ligament force can be calculated from prescribed motions of the bones relative to each other. This method was applied frequently in the 
past (Blankevoort et al., 1991; Crowninshield et al., 1976; Edwards et al., 1970; Grood and Hefzy, 1982). These studies were limited in scope, because usually the ligaments were arbitrarily represented by a limited number of line elements, disregarding their three-dimensional multibundle structures. In addition, force-displacement characteristics of the ligaments were usually estimated from literature values. Finally, the difficult problem of the zero-force length, required for these calculations, was not solved. The method was improved by Hollis et al. (1988) and Takai et al. (1993), who determined the relation between force and length for the entire ACL as well as for portions of it by the application of anterior tibial forces in different flexion angles of the knee (Takai et al., 1993) or by bone-bundle-bone testing (Hollis et al., 1988).

In order to assess the resultant force vectors and force distributions in all human knee ligaments simultaneously, a new method is presented here. This method comprises the inverse dynamics modeling approach to its full potential. In order to illustrate the utility of the new method, it was applied for the cruciate ligaments of one knee-joint specimen. The resulting loading patterns of these structures during anterior-posterior loading of the tibia at different flexion angles of the knee joint are presented and compared with literature data.

\section{METHODS AND MATERIAL}

The protocol (Fig. 1) consists of the determination of (i) the joint motions associated with externally applied anterior-posterior forces, (ii) the relationships between ligament forces and the relative positions of the bones, (iii) the insertion site geometry of bundles identified in the ligaments, (iv) the bundle force-length relationships by combining (ii) and (iii) in a separate line-element model for each ligament and, finally, (v) the forces in the bundles by combining (i), (iii) and (iv) in a whole-joint model of the knee. The protocol was applied for one cadaveric knee joint, aged 71, which was freshly frozen in a plastic bag at $-20^{\circ} \mathrm{C}$, and slowly thawed at room temperature at the time of usage. Any signs of knee pathology were absent.

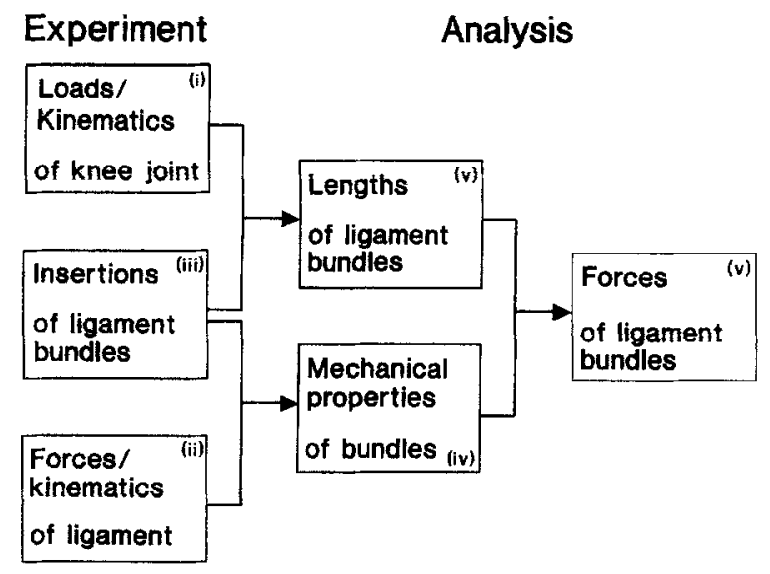

Fig. 1. Schematic diagram representing the inverse dynamics modeling approach.
To record the kinematics of the knee joint for anterior-posterior loading of the tibia (i). the femur as well as the tibia were transected at a distance of about $15 \mathrm{~cm}$ from the joint line and cemented at their ends in polymethylmethacrylate (PMMA). The fibula was fixed to the tibia with a Kirchner wire. The knee specimen was positioned in a specially designed motion and loading apparatus (Blankevoort et al., 1988). Before testing, the knee ligaments were manually preconditioned by cyclically moving the tibia through endo/exorotation, varus/valgus and anterior/posterior pathways at flexion angles of $0,15,30,60$ and $90^{\circ}$. Subsequently, the femur was fixed in flexion angles of $0,15,30,60$ and $90^{\circ}$. In the first flexion series a compressive force of $150 \mathrm{~N}$ was applied at each flexion angle. Tibial rotation was free. In subsequent flexion series, this compressive force was combined with anterior and posterior forces of $100 \mathrm{~N}$. In these series, tibial rotation angle was fixed for each flexion angle at the same value as obtained by free rotation in the first flexion series. In each flexion and loading position the relative positions of the bones were determined with Röntgen Stereophotogrammetric Analysis (RSA, Blankevoort et al., 1988; Selvik, 1974). Therefore, six tantalum pellets $(0.8 \mathrm{~mm}$ diameter $)$ were inserted in each bone before mounting the bones in the motion and loading apparatus.

To determine the relationship between ligament forces and the relative positions of the insertion sites (ii), $0.5 \mathrm{~mm}$ tantalum pellets were inserted near the insertion sites of the cruciate ligaments. The positions of the $0.5 \mathrm{~mm}$ pellets were related to the $0.8 \mathrm{~mm}$ pellets in the femur and the tibia by applying RSA. Subsequently, each ligament was isolated as a bone-ligament-bone preparation. For each bone-ligament-bone preparation a series of tensile tests was performed in which the relative orientations of the femoral and tibial insertion site were varied such that all ligament fibres were tensed at least once. The orientations of the bones were not anatomical per se. The ligament forces were measured with a load-cell. The relative insertion positions were determined with RSA. In this way, rclationships betwecn the relative positions of the bones and the ligament forces were determined (Mommersteeg et al., 1995b).

To determine the bundle insertion-site geometry (iii), obstructing bone parts and synovium were removed from the bone-ligament-bone preparations, to obtain a clear view of the fiber orientations of the ligaments from all sides. These preparations were fixed at both ends in a perspex support. The relative positions of the ligament insertions were determined by RSA. Ligament bundles were identified using the fiber orientations as a guide. In the ACL seven bundles were identified, a posterior, a posterolateral, a posteromedial, a central, an anterolateral, an anteromedial and an anterior bundle, while in the PCL six bundles were identified, a posterior or reinforcing bundle, a posterolateral, a posteromedial, an anterolateral, an anteromedial and an anterior bundle. The contour lines of the insertion sites of these bundles were measured with a 6-d.o.f. digitizer (3Space Isotrak, Polhemus Navigation Sciences, Colchester, VT, U.S.A; Mommersteeg et al., 1995c).

The force-length relationships of the fiber bundles (iv) were determined by combining the series of tensile 
tests (ii) with a model for cach ligament (Mommcrsteeg et al., 1996). In this model the bundles were represented by line elements of which the three-dimensional coordinates of the insertion sites were the geometric centers of the insertion site perimeters as measured (iii). The ligament forces were described as functions of the relative positions of the femoral and tibial insertion sites and two unknown model parameters for each line element. These unknown parameters, defining the force-length relationships of the line elements, were determined by simulating the tensile tests with the model, and subsequently, minimizing the differences in ligament forces measured in the experiment and calculated with the model for all relative positions of the bones. The ligament forces were calculated in this model as follows.

The length patterns of the line elements are determined completely by the coordinates of their insertion sites, as determined in (iii), and the three-dimensional kinematics of the femoral bone relative to the tibial one represented by the translation vector $\mathbf{D}$ and the rotation matrix $R$, as determined in (ii). The length $L_{j}$ of a line elements $j$ is calculated from the tibial insertion point $\mathbf{X}_{i j}$ relative to the tibial coordinate system and the femoral insertion point $\mathbf{x}_{f j}$ relative to the femoral coordinate system, according to

$$
L_{j}=\left|\mathbf{X}_{t j}-\mathbf{D}-R \mathbf{x}_{f j}\right| .
$$

The tensile behavior of each line element is assumed to be nonlinear elastic: the tensile force $F_{j}$ in a line element $j$ is assumed to vary according to the square of the strain $\varepsilon_{j}$ of this line element (Grood and Hefzy, 1982; Elden, 1968) as

$$
\begin{aligned}
& F_{j}=k_{j} \varepsilon_{j}^{2}, \quad \varepsilon_{j}>0, \\
& F_{j}=0, \quad \varepsilon_{j} \leqslant 0,
\end{aligned}
$$

in which $k_{j}$ is a stiffness parameter and $\varepsilon_{j}$ is the strain in line element $j$, which is calculated from its actual length $L_{j}$, as calculated in (1), and its zero force length $L_{0 j}$ according to

$$
\varepsilon_{j}=\left(L_{j}-L_{0_{j}}\right) / L_{0} .
$$

The bundle force vector $\mathbf{F}_{j}$ acting on the tibia is expressed by

$$
\mathbf{F}_{j}=F_{j} \mathbf{v}_{j},
$$

where $\mathbf{v}_{j}$ is the unit vector pointing from the tibial to the femoral insertion site of line element $j$. For the purpose of this model we assume no mutual interaction between the bundles. Under this condition, the total ligament force $F_{l}$ in each relative position of the insertion sites is found by summing the individual bundle forces $\mathbf{F}_{j}$ according to

$$
\mathbf{F}_{l}=\sum \mathbf{F}_{j} .
$$

The differences between the ligament forces measured and the corresponding values predicted by the model werc minimized by adjusting the model parameters $k_{j}$ and $L_{0 j}$, using a modification of the Levenberg-Marquardt algorithm, applying the general least-squares solver LMDIF (from MINPACK; More et al., 1980). This optimization process resulted in the identification of valucs for $k_{j}$ and $L_{0 j}$ of cach line element, and thus in their force-length relationships.

To determine the bundle forces in situ (v), the ligament multiple-bundle representations as defined in (iv) were implemented in a whole knee-joint model (Blankevoort, 1991). The three-dimensional motion characteristics of the knee-joint determined in (i) were used as input for the model. The lengths of the line elements were calculated from equation (1) for different positions of the knee. From these lengths and the parameters $k$ and $L_{0}$ (iv), the forces in the line elements were calculated from equations (2)-(5). The restraining function of a ligament (bundle) was defined as the difference in the posterior-anterior component of the bundle force between the load cases with an axial force only and those with an additional anterior-posterior force, expressed as a percentage of the externally applied load.

\section{RESULTS}

When a compressive force of $150 \mathrm{~N}$ is applied to the knee joint, the anterior cruciate ligament force decreases from about $130 \mathrm{~N}$ in extension of the joint to $0 \mathrm{~N}$ in $90^{\circ}$ of flexion (Fig. 2(a)). RSA revealed that the compressive force induced valgus and internal rotation of the tibia, combined with medial and anterior displacements. When an anteriorly directed force of $100 \mathrm{~N}$ was added, the ACL forces increased for all flexion angles with about $92 \mathrm{~N}$ maximally at $15^{\circ}$ of flexion. The percentage of the applied force restrained by the ACL varied from $70 \%$ at $15^{\circ}$ of flexion to $30 \%$ at $90^{\circ}$. This force became increasingly directed anterior-posteriorly while the knee was flexed (Fig. 3(a)). The loads in the fiber bundles varied during knee flexion and shifted along the medial side of the ligament from posterior to anterior (Fig. 3(a)). Almost uniform loading of the fiber bundles occurred between 15 and $30^{\circ}$ of flexion. At $0^{\circ}$ of flexion, $2-3 \%$ of the ACL restraint was provided by the most anterior fibers of the ligament, while at $90^{\circ}$ of flexion this percentage was $100 \%$ (Fig. 4(a)). For the most posterior bundle the reverse was true: at $0^{\circ}$ of flexion the contribution to the total ACL restraint was $67 \%$, while at $90^{\circ}$ of flexion it was zero. The lateral fiber bundles were only slightly tensed at 15 and $30^{\circ}$ of flexion.

Compressive loading of the joint resulted in minimal tension in the PCL in $0^{\circ}$ of flexion (Fig. 2(b)). When a posterior force of $100 \mathrm{~N}$ was added, the PCL force increased for all flexion angles. The resultant PCL force difference was the lowest at $30^{\circ}(13 \mathrm{~N})$ and increased towards extension $(86 \mathrm{~N})$ as well as towards higher flexion angles $(39 \mathrm{~N})$. During knee flexion, the PCL force became directed increasingly proximodistally (Fig. 3(b)). The percentage of the applied force restrained directly by the PCL was maximal in extension $(43 \%)$ and minimal at $30^{\circ}$ of flexion $(7 \%)$ (Fig. $2(\mathrm{~b})$ ). This restraint was provided completely by the posterior and posterolateral bundles (Fig. 4(b)). At higher flexion angles, the more anteriorly positioned bundles became the primary restraints, in particular the anteromedial bundles: at $60^{\circ}$ of flexion, the contributions of the more anteriorly vs the more posteriorly positioned bundles to the total PCL restraint were 48 and $52 \%$, respectively, while at $90^{\circ}$ of flexion. they were 92 and $8 \%$, respectively. 

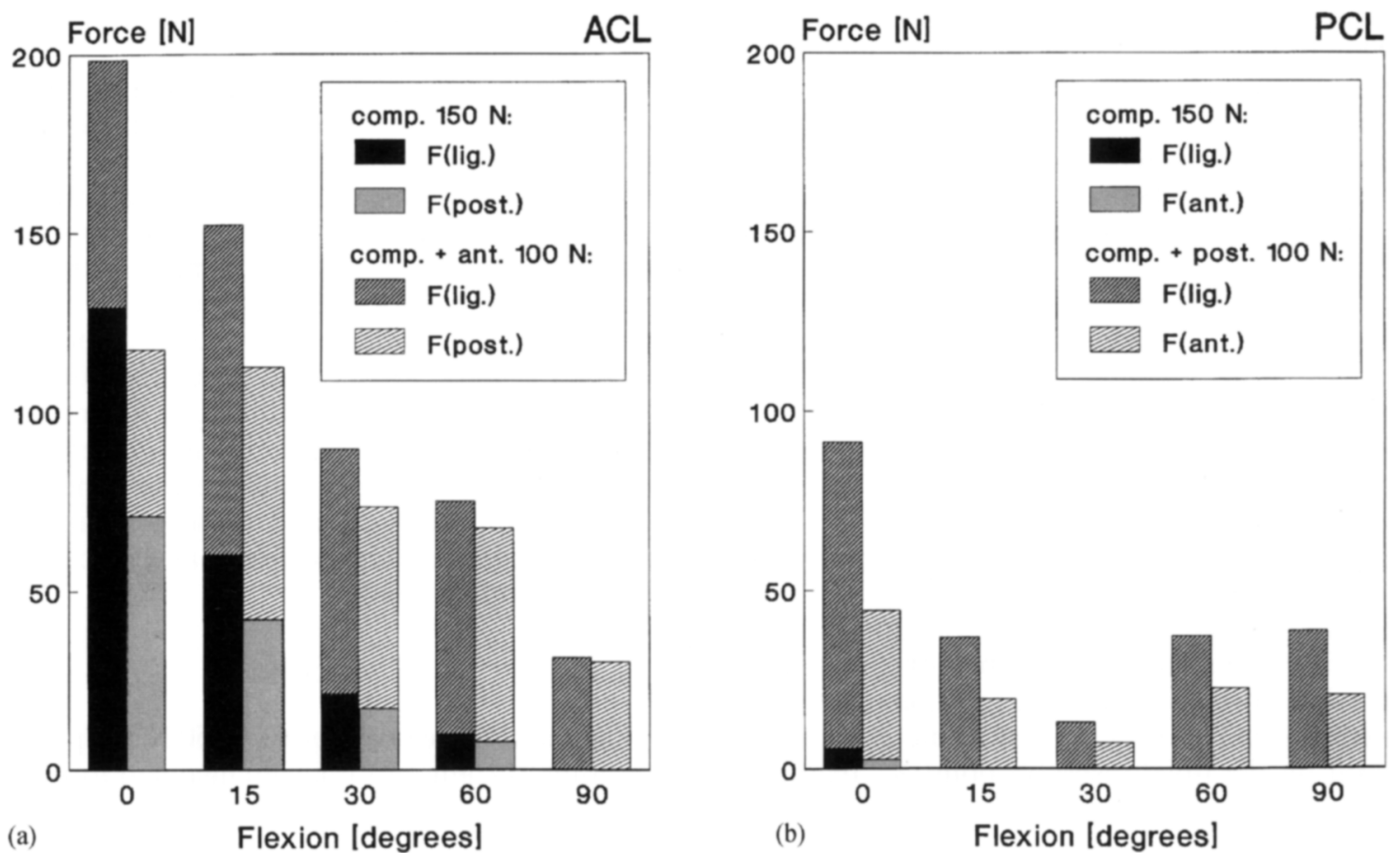

Fig. 2. Resultant forces (first column of stacked bars) as well as the anterior-posterior components of these forces (second column of stacked bars) of the ACL (a) and the PCL (b) at $0,15,30,60$ and $90^{\circ}$ of flexion with an externally applied compressive force of $150 \mathrm{~N}$ (black and grey) in combination with anterior and posterior forces of $100 \mathrm{~N}$, respectively (cross-hatched).

\section{DISCUSSION}

In this study it was shown that with an inverse dynamics approach it is feasible to obtain detailed threedimensional load-distributions of knee ligaments simultaneously. The preliminary results for one knee-joint specimen confirmed the complexity of load transfer through the cruciate ligaments, whereby each part of a ligament plays a characteristic role in particular phases of knee motion. A gradual transition of forces is found from the posterior fiber bundles to the anterior ones. Hence, the contributions of the ligaments to knee restraint depend strongly on its flexion angle.

The methods used have some unique features. The forces in several bundles of more than one ligament can be determined simultaneously. Magnitudes, directions and points of application of these forces can be determined. The methods imply determination of the in situ ligament bundle lengths from kinematic data. The parametric characterization of ligament behavior in whole ligament tests, for different relative insertion orientations, provides the load-length curves of the bundles. In situ loads are obtained by simply comparing the bundle lengths with their load-length curves, in each relevant situation. The way in which the parameters describing the force-length relationships of the ligament bundles are identified tackles two problems common in ligament testing. First, ligament properties have been shown to depend on the alignment of the ligament in the testing machine (Woo et al., 1991 and others). With the present method, the test orientations are varied and the variable ligament behavior is accounted for in the analysis. Sec- ond, it offers a solution for the problem of the zero-load length determination of the ligament (bundles).

Another feature characteristic for the new method is that the multiple-line representations of the ligaments are based on actual measurements of the anatomy. In the past, ligaments were modeled by two or three arbitrary line elements, based on measurements of the ligament insertion sites' perimeters (Blankevoort, 1991; Van Dijk, 1983; Takai et al., 1993). Blankevoort (1991) showed that two or three line elements do not suffice to stabilize the knee during its entire range of motion. Hence, the present method is expected to produce more precise and anatomically consistent information.

In comparison with the direct measuring approach, the method described here has the advantage that no external devices must be attached to the ligament which might cause impingement problems or alterations in the load-elongation behavior of the ligament. Furthermore, the joint remains intact during the kinematic experiment, preserving the anatomic relationships within the joint. In dissection studies, the removal of a supporting structure might influence the internal configuration of remaining knee-joint structures and, thus, their role in knee-joint mechanics. The ligaments provide direct restraints to knee-joint motion, but also indirect articular restraints caused by interaction between ligaments and the articular surfaces (Huiskes and Blankevoort, 1991). In addition there are interactions between a ligament and other knee structures, such as the menisci, the capsule and other ligaments which provide also indirect restraints. Using the present method, the direct individual ligament restraint is determined in isolation, while in dissection 

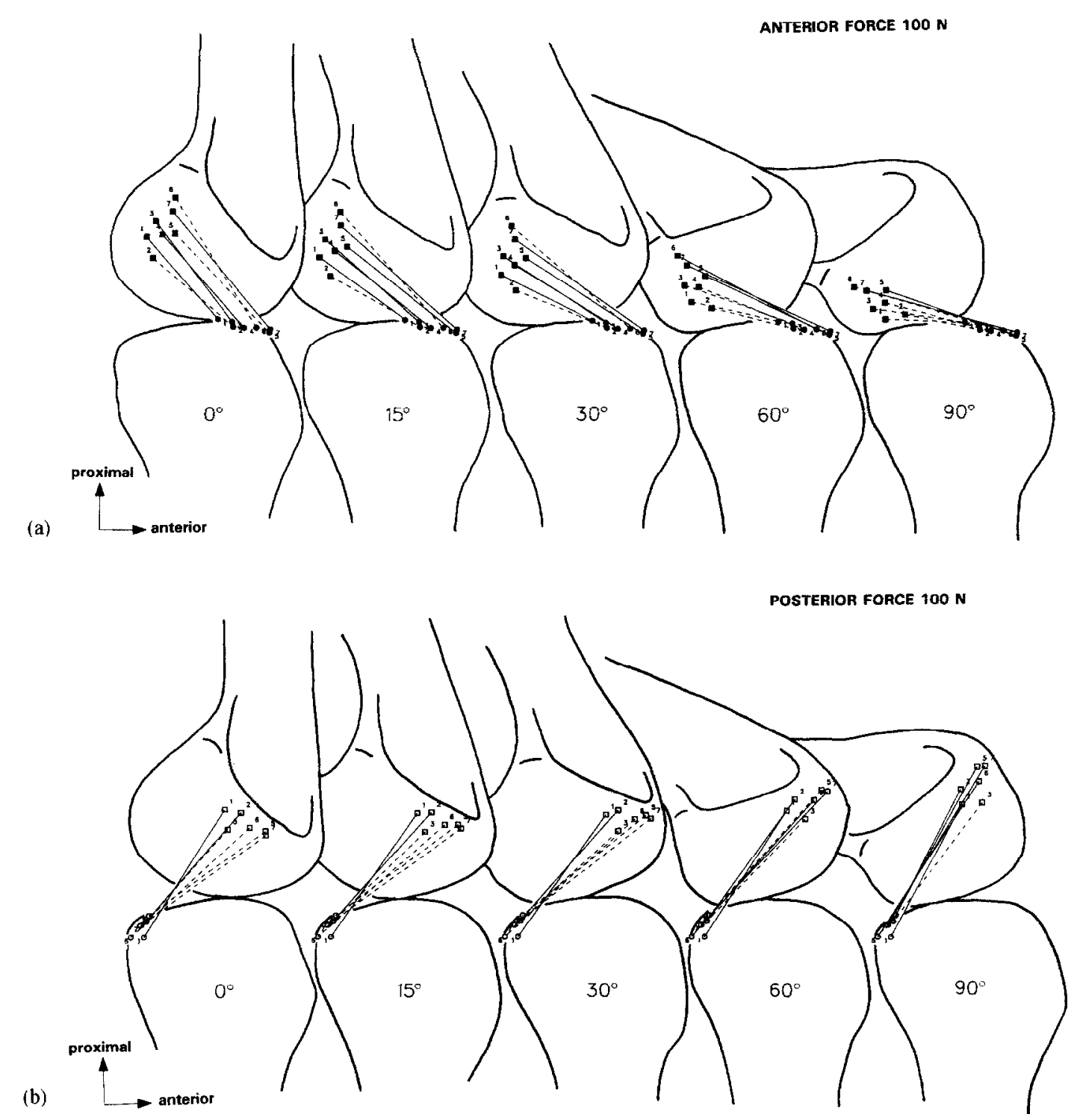

Fig. 3. Medial views of the line element representations of the ACL (a) and the PCL (b) in situ at $0.15,30,60$ and $90^{\circ}$ of flexion with an anterior and posterior force of $100 \mathrm{~N}$, respectively. A loaded line element is presented by a continuous line and a nonloaded line element by a dotted line. The femoral insertion sites of these line elements are presented by square markers and the tibial insertion sites by round markers. Legends: Fiber bundles: 1. posterior, 2. posterolateral, 3. posteromedial, 4. central, 5. anterolateral, 6 . anteromedial, 7. anterior.

studies the indirect effects are also included. The method is not limited with respect to the complexity of the knee loading configuration and the degrees of freedom of the test as is the case in dissection studies in which the maintenance of the path of motion before and after dissection of a structure is a prerequisite (Blomstrom et al., 1993; Takai et al., 1993; Vahey and Draganich, 1991).

However, our approach carries practical limitations. The experimental technique applied is extremely time consuming and technologically involved. Secondly, the ligaments are represented by a number of line elements which do not interact with each other. It was assumed that the effects of mechanical bundle-matrix and interbundle interactions are negligible. For the ACL it was demonstrated that longitudinal separation of the anterior and posterior portions of the $A C L$ influences the stiffness of the knee in anterior tibial loading only minimally (Blomstrom et al., 1993; Takai et al., 1993). However, it could be a factor which affects the accuracy of our results. Furthermore, it is assumed that the multi-lineelement models can extrapolate information to other ligament orientations which occur in situ, but were not represented in the bench tests. Implicitly, it is assumed that the bundles are small enough to assume their force-length relationships independent of the relative orientations of the bones. Some of the uncertainties inherent to these limitations, however, have been removed by a global validation study of the ligament models, recently performed in our laboratory (Mommersteeg et al., 1995a). Of course, these limitations are not inherent to our approach in general, because the multi line element 

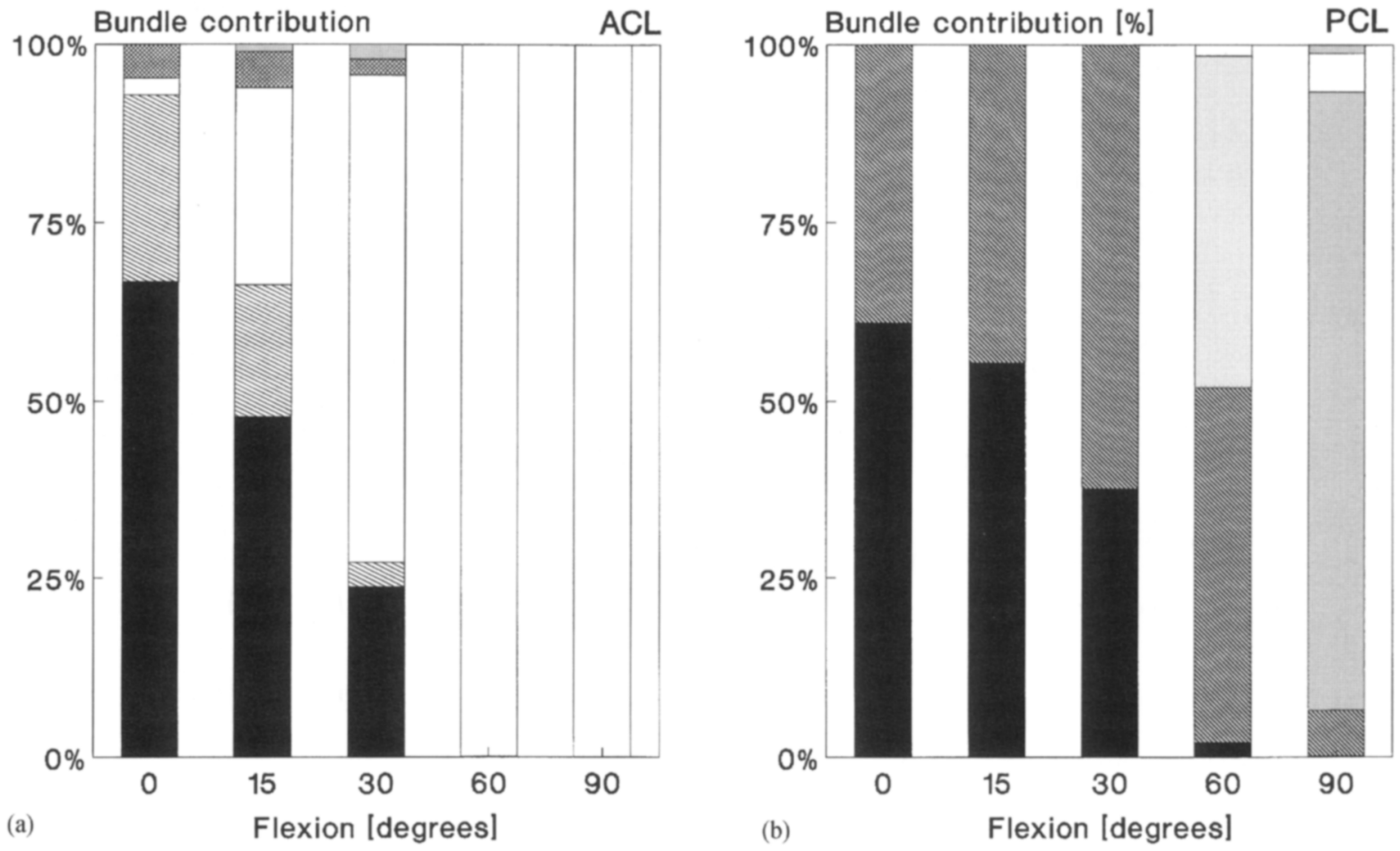

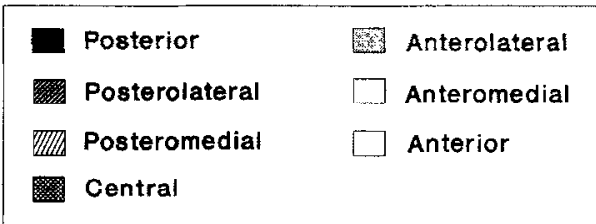

Fig. 4. The contributions of the ligament bundles to the ACL-restraint to anterior loading (a) and to the PCL-restraint to posterior loading (b) at flexion angles of $0,15,30,60$ and $90^{\circ}$ of flexion. The black bars present fiber bundles at the postcrior side of the ligaments. Toward the anterior side of the ligaments, the bars turn gradually into white bars.

models could also be replaced by more precise threedimensional solid models in the future.

Another limitation not inherent to the method per se, but to the way in which it was applied here is related to the relevance of the results to the in vivo situation. To represent in vivo conditions, realistic loads must be represented in the knee tests. In our experimental design, this is possible only to a certain extent. In particular, the compressive kncc-joint forces we applicd were limited to about $150 \mathrm{~N}$. The results are strongly dependent on the compressive force applied. The compressive force influences the distribution of restraints between the articular contact areas and the ligaments. With a compressive force, the contribution of the contact becomes higher (Hsieh and Walker, 1976). Under $925 \mathrm{~N}$ of tibio-femoral contact force, the resultant ACL force was decreased as much as $36 \%$ at $0^{\circ}$ of flexion and $46 \%$ at $20^{\circ}$ of flexion (Markolf et al. 1991).

There is general consensus in the literature that the $\mathrm{ACL}$ and the PCL are the primary structures resisting anterior and posterior tibial loading, respectively (Ahmed et al., 1987; Blankevoort, 1991; Butler et al., 1980;
Markolf et al., 1991; Piziali et al., 1980; Wascher et al., 1993). This was once more confirmed in our approach. The actual force patterns we found in the PCL during posterior loading of the tibia, however, are not compatible with the results of Wascher et al. (1993). At a $50 \mathrm{~N}$ posterior tibial force, the mean PCL force was minimum at extension and increased gradually towards $58.4 \mathrm{~N}$ at $90^{\circ}$ of flexion. The force patterns are, however, similar to those of Race and Amis (1993), who showed that the PCL force peaked twice, at extension and at $70^{\circ}$ of flexion, for 2, 4 and $6 \mathrm{~mm}$ posterior tibial translations. Vahey and Draganich (1991) also found an increase in PCL force from 30 to $90^{\circ}$ of flexion.

At a compressive force of $150 \mathrm{~N}$ only, the ACL forces decrease from $130 \mathrm{~N}$ in extension to $0 \mathrm{~N}$ at $90^{\circ}$ of flexion. Markolf et al. (1991) measured considerable variation in the magnitudes of ACL force at passive knee extension. At $5^{\circ}$ hyperextension, forces varied from 50 to $241 \mathrm{~N}$ and at $0^{\circ}$ of fiexion from 16 to $87 \mathrm{~N}$. From $20^{\circ}$ of flexion ACL forces were zero. When a $200 \mathrm{~N}$ quadriceps tendon pull was applied, resulting in compressive and anteriorly directed forces in the joint, the ACL forces became similar 
to those found in the present study. With anterior tibial loading, the increases in ACL force were lower than the applied anterior tibial load for all flexion angles in the present study, with a maximal increase at $15^{\circ}$ of flexion $(92 \mathrm{~N})$. This corresponds to the results of Vahey and Draganich (1991) and Ahmed et al. (1987). Takai et al. (1993) and Markolf et al. (1991), however, found that the resultant forces in the ACL were slightly higher than the anteriorly directed forces applied externally. Our finding that the loads in the ACL are more uniform at lower than at greater flexion angles is in agreement with Takai et al. (1993), who found a contribution of $70 \%$ of the anterior part of the ligament at $30^{\circ}$ of flexion, which decreases towards extension $(47 \%)$ and increases towards $90^{\circ}$ flexion $(93 \%)$. That the lateral bundle of the ACL does not play a significant role in resisting anterior tibial loading is in agreement with the findings of Hollis et al. (1988) and Blomstrom et al. (1993).

It appeared that relatively high forces during functional loading are carried by only a few fiber bundles. Therefore, the maximum stress that exists in a ligament cannot be determined by dividing the total force carried by the ligament by its cross-sectional area. Concentrations of the force in only a few fiber bundles of the ligament can result in (micro)failures at loads below those which would cause a ligament which is uniformly stressed to fail.

Data as obtained in the present study can be useful for the design of improved ligament reconstructions. The preliminary results suggest that the function of the ligaments, with these unique three-dimensional multibundle arrangements, may be difficult to reconstruct with a single-stranded structure. It may be more appropriate to reconstruct the ligaments with multiple strands, each with its own function at different flexion angles. There have been reports from studies indicating that the use of two-stranded reconstructions provided better results (Radford and Amis, 1990; Zaricznyj, 1987).

The information provided with an experimental approach as presented here is also useful for the evaluation of knee-ligament injuries, particularly for isolated lesions of fiber bundles. Two different tests are popular for the evaluation of anterior cruciate ligament lesions. In $90^{\circ}$ of flexion the anterior drawer test is performed and in $15^{\circ}$ of flexion the Lachman test. Our preliminary results suggest that during an anterior drawer test only the most anterior bundle is tested. An isolated lesion of this bundle could be detected in this way. With a Lachman test, the fiber bundles of the ACL are loaded more uniformly.

In conclusion, the approach presented here, involving inverse dynamics, whole knee-joint and ligament computer models, with associated experimental tests, is suitable to estimate detailed load distributions in the (kneejoint) ligaments simultaneously, in a variety of motion and loading conditions. Application to other joints is also possible. The information provided is unique and useful, albeit the method is elaborate and extremely time consuming. Evidently, where it concerns the present application to the cruciate ligaments, more knee specimens must be tested to verify the observed force distributions among the several fiber bundles during knee flexion.
Acknowledgements - We like to thank Willem van de Wijdeven and Patrick Maathuys for their assistance during the experiments.

\section{REFERENCES}

Ahmed, A. M., Burke, D. L., Duncan, N. A. and Chan, K. H. (1992) Ligament tension pattern in the flexed knee in combined passive anterior translation and axial rotation. J. orthop. Res. 10, 854-867.

Ahmed, A. M., Hyder, A., Burke, D. L. and Chan, K. H. (1987) In vitro ligament tension pattern in the flexed knce in passive loading. J. orthop. Res. 5, 217-230.

Blankevoort, L. (1991) Passive motion characteristics of the human knee joint: experiments and computer simulations. Dissertation, University of Nijmegen, Nijmegen, The Netherlands.

Blankevoort, L., Huiskes, R. and Lange, A. de (1988) The envelope of passive knee joint motion. J. Biomechanics 21, 705-720.

Blankevoort, L., Huiskes, R. and Lange, A. de (1991) Recruitment of knee-joint ligaments. $J$ Biomechanics 113, 94-103.

Blomstrom, G. L., Livesay, G. A., Fujie, H., Smith, B. A., Kashiwaguchi, S. and Woo, S. L.-Y. (1993) Distribution of in situ forces within the human anterior cruciate ligament. Am. Soc. Mech. Engny AMD 24, 359-362.

Butler, D. L., Noyes, F. R. and Grood, E. S. (1980) Ligamentous restraints to anterior-posterior drawer in the human knee. $J$. Bone $J_{t}$ Surg. 62, 259-270.

Crowninshield, R., Pope, M. H. and Johnson, R. J. (1976) An analytical model of the knee. J. Biomechanics 9, 397-405.

Dijk, R. van (1983) The behaviour of the cruciate ligaments in the human knee.' Dissertation, University of Nijmegen, Nijmegen, The Netherlands.

Edwards, R. G., Lafferty, J. F. and Lange, K. O. (1970) Ligament strain in the human knee joint. J. basic Engng 92, 131-136.

Elden, H. R. (1968) Physical properties of collagen fibers. In: International Review of Connective Tissue Research, Vol. 24 (Edited by Hall, D. A.), pp 283-348. Academic Press. New York.

Grood, E. S. and Hefzy, M. S. (1982) An analytical technique for modeling knee joint stiffness-part $\mathrm{I}$ : ligamentons forces. I. hiomech. Engng 104, 330-337.

Guan, Y., Butler, D. L., Dormer, S. G. and Cummings, J. F. (1991) Contribution of anterior cruciate ligament subunits during anterior drawer in the human knee. Trans. orthop. Res. Soc. 16, 239.

Hollis, J. M., Marcin, J. P., Horibe, S. and Woo, S. L.-Y. (1988) Load determination in $\mathrm{ACL}$ fiber bundles under knee loading. Trans. orthop. Res. Soc. 13, 196.

Hsieh, H. and Walker, P. (1976) Stabilizing mechanisms of the loaded and unloaded knee joint. J. Bone Jt Surg. [Am] 58, 87-93.

Huiskes, R. (1992) Mathematical modeling of the knee. In: Biology and Biomechanics of the Traumatized Synovial Joint: the Knee as a Model, Chapter 21 (Edited by Finerman, G. A. M. and Noyes, F. R.). American Orthopaedic Society for Sports Medicine, Rosemont, IL.

Huiskes, R. and Blankevoort, L. (1991) The relationship between knee motion and articular surface geometry. In: Biomechanics of Diarthrodial Joints, Vol. II (Edited by Mow, V. C., Ratcliffe, A. and Woo, S. L.-Y.), pp. 269-286. Springer, New York.

Lewis, J. L., Lew, W. D., Hill, J. A., Hanley, P., Ohland, K., Kirstukas, S. and Hunter, R. E. (1989) Knee joint motion and ligament forces before and after ACL reconstruction. J. biomech. Engng 111, 97-106.

Lewis, J. L., Lew, W. D. and Schmidt, J. (1982) A note on the application and evaluation of the Buckle transducer for knee ligament force measurement. J. biomech. Engng 104, 125-128.

Markolf, K. L., Gorek, J. F., Kabo, J. M., Shapiro, M.S. and Finerman, G. A. M. (1991) New insights into load bearing functions of the anterior cruciate ligament. In: Biomechanics of Diarthrodial Joints, Vol. I (Edited by Mow, V. C., Ratcliffe, A. and Woo. S. L.-Y.) pp. 155-175. Springer, New York.

Markolf, K. L., Wascher, D. C. and Finerman, G. A. M. (1993) Direct in vitro measurement of forces in the cruciate ligaments. Part II: The effect of section of the posterolateral structures. J. Bone Ji Surg. [Am] 75(3), 387-394.

Mommersteeg, T. J. A., Blankevoort, L. and Huiskes, R. (1995a) A global verification of human knee ligament models. Poster presented at the 41st Annual Orthopaedic Research Society, Orlando, U.S.A. 294. Mommersteeg, T. J. A., Blankevoort, L., Huiskes, R., Kooloos, J. G. M. and Kauer, J. M. G. (1996) Characterization of the mechanical behavior of human knee ligaments. J. Biomechanics 29, 151-160. 
Mommersteeg, T. J. A., Blankevoort, L., Huiskes, R., Kooloos, J. G. M., Kauer, J. M. G. and Hendriks, J. C. M. (1995b) The effect of variable relative insertion orientation of human knee bone-ligament-bone complexes on the tensile stiffness. $J$. Biomechanics 28, 745-752.

Mommersteeg, T. J. A., Kooloos, J. G. M., Blankevoort, L., Kauer, J. M. G.. Huiskes, R. and Roeling, F. Q. C. (1995c) The fbre bundle andomy of human cruciate ligaments. $J$. Anat. 187, 461-471.

Moré, J., Garbow, B. and Hillstrom, K. (1980) User guide for MINPACK-1, Argonne National Labs Report $\Lambda$ NL-80-74, rgonne, ILL

Piziali, R. L., Seering. W. P., Nagel, D. A. and Schurman, D. J. (1980) The function of the primary ligaments of the knee in anterior-posterior and medial-lateral motions. J. Biomechanics 13, 777-784.

Race, A. and Amis, A. A. (1993) Loading of the two bundles of the posterior cruciate ligament: an analysis of bundle function in AP-drawer. Proc. 30th Conf. of the European Orthopaedic Research Society, 67.

Radford. W. J. P. and Amis, A. A. (1990) Biomechanics of a double prosthetic ligament in the anterior cruciate deficient knee. I. Bone.It Surg. 72B(6), 1038-1043.

Selvik, G. (1974) A Röntgenstereophotogrammetric method for the study of the kinematics of the skclctal system. Ph.D. Thesis, Univer- sily of Lund, Sweden. Reprinted as: Röntgen Stereophotogrammetry - a method for the study of the kinematics of the skeletal system (1989). Acta orthop. scand. (Suppl. 232), Vol. 60. 1-51.

Takai, S., Adams, D. J., Livesay, G. A. and Woo, S. L.-Y. (1993) Determination of the in situ loads on the human anterior cruciate ligament. J. orthop. Res. 11, 686-695.

Vahey, J. W. and Draganich, L. F. (1991) Tensions in the anterior and posterior cruciate ligaments of the knee during passive loading: predicting ligament loads from in situ measurements. J. orthop. Res 9, 529-538

Wascher, D. C., Markolf, K. L., Shapiro, M. S. and Finerman, G. A. M. (1993) Direct in vitro measurement of forces in the cruciate ligaments. Part I: The effect of multiplane loading in the intact knee. $J$. Bone $J t$ Surg. [Am] 75(3), 377-386.

Woo, S. L.-Y., Hollis, J. M., Adams, D. J., Lyon, R. M. and Takai, S (1991) Tensile properties of the human femur anterior cruciate ligament-tibia complex: the effects of specimen age and orientation. Am. J. Sports Med. 19(3), 217-225.

Zaricznyj, J. B. (1987) Reconstruction of the anterior cruciate ligament of the knee using a doubled tendon graft. Clin. Orthop. 220, 162-175. 